\title{
A New Form of Path Integral for the Coherent States Representation and its Semiclassical Limit
}

\author{
L. C. dos Santos and M. A. M. de Aguiar \\ Instituto de Física 'Gleb Wataghin' \\ Universidade Estadual de Campinas, Unicamp \\ 13083-970, Campinas, São Paulo, Brasil
}

Received on 18 November, 2004

\begin{abstract}
The overcompleteness of the coherent states basis leads to a multiplicity of representations of Feynman's path integral. These different representations, although equivalent quantum mechanically, lead to different semiclassical limits. Two such semiclassical formulas were derived in [1] for the two corresponding path integral forms suggested by Klauder and Skagerstan in [2]. Each of these formulas involve trajectories governed by a different classical representation of the Hamiltonian operator: the $\mathrm{P}$ representation in one case and the Q representation in other. In this paper we construct a third representation of the path integral whose semiclassical limit involves directly the Weyl representation of the Hamiltonian operator, i.e., the classical Hamiltonian itself.
\end{abstract}

\section{Introduction}

In recent years there has been a renewed interest in semiclassical approximations with coherent states. These approximations have found applications in many areas of physics and chemistry. The semiclassical coherent state propagator has a long history, that starts with Klauder $[3,4,5]$ and Weissman [6]. Several properties of the propagator were subsequently studied for a number of fundamental quantum processes (see, e.g.,[7-17]). More recently, a detailed derivation of the semiclassical propagator for systems with one degree of freedom was presented in [1].

The set of coherent states forms an non-orthogonal overcomplete basis, since each state in the set can be written as a linear combination of the others. This overcompleteness, on the other hand, has important consequences in the path integral formulation of the propagator. It implies in the existence of several forms of the path integrals, all equivalent quantum mechanically, but each leading to a slightly different semiclassical limit. Klauder and Skagerstam [2] proposed two basic forms for the coherent state path integral, each of them having their corresponding advantages and problems [2]. The semiclassical limit of these two basic propagators were considered in [1] where it was shown that both propagators can written in terms of classical complex trajectories, each governed by different classical representation of the Hamiltonian operator $\hat{H}$ : the P representation in one case and the Q representation in other. We briefly review these representations in section 2 . The phase appearing in these semiclassical formulas turns out to be not just the action of the corresponding complex classical trajectory, but it also contains a 'correction term' $I$ that comes with different signs in each formula (see Eqs.(15) and (16)).

In [1] it was also suggested that a semiclassical represen- tation involving directly the Weyl representation of $\hat{H}$, or the classical Hamiltonian, could probably be constructed, and a formula for this representation was conjectured. In this paper we derive this formula by constructing a new representation of the quantum mechanical path integral, Eq.(26), and deriving its semiclassical limit Eq.(51). We show that the classical trajectories involved in this formula are governed by the average between the $\mathrm{P}$ and $\mathrm{Q}$ representations of the Hamiltonian operator. The correction term in the phase, on the other hand, turns out to be one half of the difference between the corresponding terms in the previous formulations. We then show that this average Hamiltonian can be replaced by the classical one and the correction term discarded, the error being of order $\hbar^{2}$. Our final result is the conjectured formula, Eq.(57).

This paper is organized as follows: in section 2 we review the path integral constructions of Klauder and Skagerstan [2] and their semiclassical approximations [1]. In section 3 we construct the new quantum representation and derive its semiclassical limit. Finally in section 4 we show that, within the validity of the approximations, this formula coincides with the equation suggested in [1].

\section{The coherent state propagator and its semiclassical approximations}

\subsection{The propagator}

The coherent state $|z\rangle$ of a harmonic oscillator of mass $m$ and frequency $\omega$ is defined by

$$
|z\rangle=\mathrm{e}^{-\frac{1}{2}|z|^{2}} \mathrm{e}^{z \hat{a}^{\dagger}}|0\rangle
$$


with $|0\rangle$ the harmonic oscillator ground state and

$$
\hat{a}^{\dagger}=\frac{1}{\sqrt{2}}\left(\frac{\hat{q}}{b}-i \frac{\hat{p}}{c}\right), \quad z=\frac{1}{\sqrt{2}}\left(\frac{q}{b}+i \frac{p}{c}\right) .
$$

In the above $\hat{q}, \hat{p}$, and $\hat{a}^{\dagger}$ are operators; $q$ and $p$ are real numbers; $z$ is complex. The parameters $b=(\hbar / m \omega)^{\frac{1}{2}}$ and $c=(\hbar m \omega)^{\frac{1}{2}}$ define the length and momentum scales, respectively, and their product is $\hbar$.

For a time-independent Hamiltonian operator $\hat{H}$, the propagator in the coherent states representation is the matrix element of the evolution operator between the states $\left|z^{\prime}\right\rangle$ and $\left|z^{\prime \prime}\right\rangle$ :

$$
K\left(z^{\prime \prime}, T ; z^{\prime}, 0\right)=\left\langle z^{\prime \prime}\left|\mathrm{e}^{-\frac{i}{\hbar} \hat{H} T}\right| z^{\prime}\right\rangle
$$

We restrict ourselves to Hamiltonians that can be expanded in a power series of the creation and annihilator operators $\hat{a}^{\dagger}$ and $\hat{a}$.

In the derivation the semiclassical limit of the propagator, the Hamiltonian operator $\hat{H}$ is somehow replaced by a classical Hamiltonian function $H(q, p)$. This 'replacement', however, is not uniquely defined, and the ambiguities that exist in the relation between the operator $\hat{H}$ and the function $H(q, p)$ also arise in connection with the overcompleteness of the coherent state basis, as we shall see in the next subsections.

There are actually many ways to associate a classical function of position and momentum $A(q, p)$ to a quantum mechanical operator $\hat{A}$. However, three of them are specially important. The first one, denoted $A_{Q}(q, p)$, is called the $\mathrm{Q}$ representation of the operator $\hat{A}$ and is constructed as follows: one writes $\hat{A}$ in terms of the creation and annihilation operators $\hat{a}^{\dagger}$ and $\hat{a}$ in such a way that all the creation operators appear to the left of the annihilation operators, making each monomial of $\hat{A}$ look like $c_{n m} \hat{a}^{\dagger n} \hat{a}^{m}$. Then we replace $\hat{a}$ by $z$ and $\hat{a}^{\dagger}$ by $z^{\star}$. The inverse of this operation, that associates a quantum operator to a classical function, is called 'normal ordering'. In this case one first writes the classical function in terms of $z$ and $z^{\star}$, with all the $z^{\star}$ 's to the left of the $z$ 's, and then replace $z$ by $\hat{a}$ and $z^{\star}$ by $\hat{a}^{\dagger}$.

The second possibility, called the P representation of $\hat{A}$, is obtained by a similar procedure, but this time the monomials of $\hat{A}$ are written in the opposite order, such that they look like $c_{n m} \hat{a}^{n} \hat{a}^{\dagger m}$. Once the operator has been put in this form one replaces again $\hat{a}$ by $z$ and $\hat{a}^{\dagger}$ by $z^{\star}$ to obtain $A_{P}(q, p)$. The inverse of this operation is called 'anti-normal ordering'. Notice that the differences between the two representations come from the commutator of $\hat{q}$ and $\hat{p}$, which is proportional to $\hbar$. Therefore, these differences go to zero as $\hbar$ goes to zero.

There is, finally, a third representation which is the most symmetric of all, and therefore the most natural. It is given by the Wigner transformation

$$
A_{W}(q, p)=\int \mathrm{d} s \mathrm{e}^{\frac{i}{\hbar} p s}\left\langle q-\frac{s}{2}|\hat{A}| q+\frac{s}{2}\right\rangle .
$$

$A_{W}(q, p)$ is called the Weyl representation of $\hat{A}[18,19]$. Its inverse transformation consists in writing the classical function in terms of $z$ and $z^{\star}$ considering all possible orderings for each monomial and making a symmetric average between all possibilities before replacing $z$ and $z^{\star}$ by the corresponding operators. As an illustration of these three representations we take

$$
\hat{H}=\frac{1}{2} \frac{\partial^{2}}{\partial x^{2}}+\frac{1}{2} x^{2}+x^{4}
$$

$(m=\hbar=1)$ for which we obtain

$$
\begin{aligned}
& H_{Q}=\frac{1}{2}\left(p^{2}+x^{2}\right)+x^{4}+\frac{1}{4}\left(b^{2}+b^{-2}\right)+3 b^{2} x^{2}+3 b^{4} / 4 \\
& H_{P}=\frac{1}{2}\left(p^{2}+x^{2}\right)+x^{4}-\frac{1}{4}\left(b^{2}+b^{-2}\right)-3 b^{2} x^{2}+3 b^{4} / 4 \\
& H_{W}=\frac{1}{2}\left(p^{2}+x^{2}\right)+x^{4}
\end{aligned}
$$

where $b$ is the width of the coherent state. Notice the term proportional to $x^{2}$ that appears with opposite signs in $H_{Q}$ and $H_{P}$, really modifying the classical dynamics with respect to $H_{W}$.

In the next subsections we shall see how these different representations appear naturally in the semiclassical limit of the coherent state propagator.

\subsection{Basic Path Integrals and their Semiclassi- cal Approximations}

The calculation of the semiclassical propagator in the coherent state representation starting from path integrals was discussed in detail in [1]. In this section we summarize these previous results emphasizing the non-uniqueness of the semiclassical limit as a consequence of the overcompleteness of the coherent state representation. The reader is referred to [1] for the details.

In order to write a path integral for $K\left(z^{\prime \prime}, T ; z^{\prime}, 0\right)$, the time interval has to be divided into a large number of slices and, for each slice, an infinitesimal propagator has to be calculated. As pointed out by Klauder and Skagerstam [2], there are at least two different ways to do that. Each of these gives rise to a different representation of the path integral. Although they correspond to identical quantum mechanical quantities, their semiclassical approximations are different. We review the construction of these two representations below.

The first form of path integral is constructed by breaking the time interval $T$ into $N$ parts of size $\tau$ and inserting the unit operator

$$
\mathbb{1}=\int|z\rangle \frac{\mathrm{d}^{2} \mathrm{z}}{\pi}\langle z|
$$

everywhere between adjacent propagation steps. We denote the real and imaginary parts of $z$ by $x$ and $y$, respectively. In all integrations, $\mathrm{d}^{2} z / \pi$ means $\mathrm{d} x \mathrm{~d} y / \pi$. After the insertions, the propagator becomes a $2(N-1)$-fold integral over the whole phase space 


$$
K\left(z^{\prime \prime}, t ; z^{\prime}, 0\right)=\int\left\{\prod_{j=1}^{N-1} \frac{\mathrm{d}^{2} z_{j}}{\pi}\right\} \prod_{j=0}^{N-1}\left\{\left\langle z_{j+1}\left|\mathrm{e}^{-\frac{i}{\hbar} \hat{H}\left(t_{j}\right) \tau}\right| z_{j}\right\rangle\right\}
$$

with $z_{N}=z^{\prime \prime}$ and $z_{0}=z^{\prime}$. Using the coherent state overlap formula

$$
\left\langle z_{j+1} \mid z_{j}\right\rangle=\exp \left\{-\frac{1}{2}\left|z_{j+1}\right|^{2}+z_{j+1}^{\star} z_{j}-\frac{1}{2}\left|z_{j}\right|^{2}\right\}
$$

and expanding $e^{-i H \tau / \hbar} \approx 1-i H \tau / \hbar$ we write

$$
\left\langle z_{j+1}\left|\mathrm{e}^{-\frac{i}{\hbar} \hat{H}\left(t_{j}\right) \tau}\right| z_{j}\right\rangle=\exp \left\{\frac{1}{2}\left(z_{j+1}^{\star}-z_{j}^{\star}\right) z_{j}-\frac{1}{2} z_{j+1}^{\star}\left(z_{j+1}-z_{j}\right)-\frac{i \tau}{\hbar} \mathcal{H}_{j+1, j}\right\}
$$

where

$$
\mathcal{H}_{j+1, j} \equiv \frac{\left\langle z_{j+1}\left|\hat{H}\left(t_{j}\right)\right| z_{j}\right\rangle}{\left\langle z_{j+1} \mid z_{j}\right\rangle} \equiv \mathcal{H}\left(z_{j+1}^{\star}, z_{j} ; t_{j}\right)
$$

and $\left(1-i \mathcal{H}_{j+1, j} \tau / \hbar\right)$ has been approximated again by $e^{-i \mathcal{H}_{j+1, j} \tau / \hbar}$. With these manipulations the first form of the propagator, that we shall call $K_{1}$, becomes

$$
K_{1}\left(z^{\prime \prime}, t ; z^{\prime}, 0\right)=\int\left\{\prod_{j=1}^{N-1} \frac{\mathrm{d}^{2} z_{j}}{\pi}\right\} \exp \left\{\sum_{j=0}^{N-1}\left[\frac{1}{2}\left(z_{j+1}^{\star}-z_{j}^{\star}\right) z_{j}-\frac{1}{2} z_{j+1}^{\star}\left(z_{j+1}-z_{j}\right)-\frac{i \tau}{\hbar} \mathcal{H}_{j+1, j}\right]\right\}
$$

When the limit $N \rightarrow \infty$ (respectively $\tau \rightarrow 0$ ) is taken, the above summations turn into integrals, and expressions (10) appears to be exact, were it not for the well-known problems attached to the meaning of such functional integrals. Also, $\mathcal{H}_{j+1, j}$ turns into the smooth Hamiltonian function $\mathcal{H}\left(z, z^{\star}\right) \equiv H_{1}\left(z, z^{\star}\right) \equiv\langle z|\hat{H}| z\rangle$. Using the properties $\hat{a}|z\rangle=z|z\rangle$ and $\langle z| \hat{a}^{\dagger}=\langle z| z^{\star}$, we see that $\mathcal{H}$ can be easily calculated if $\hat{H}$ is written in terms of creation and annihilation operators with all $\hat{a}^{\dagger}$ 's to the left of the $\hat{a}$ 's. Therefore, $\mathcal{H}$ is exactly the $\mathrm{Q}$ symbol of the Hamiltonian operator [18].

The second form of path integral starts from the "diagonal representation" of the hamiltonian operator, namely

$$
\hat{H}=\int|z\rangle H_{2}\left(z^{\star}, z\right) \frac{\mathrm{d}^{2} z}{\pi}\langle z| .
$$

Assuming that $\hat{H}$ is either a polynomial in $p$ and $q$ or a con- verging sequence of such polynomials, this diagonal representation always exists. The calculation of $\mathrm{H}_{2}$ is not as direct as that of $H_{1}$, but it can be shown [18] that $H_{2}\left(z^{\star}, z\right)$ is exactly the $\mathrm{P}$ symbol of $\hat{H}$. This second form will be contrasted with the first-form hamiltonian function $H_{1}\left(z^{\star}, z\right)$. To facilitate the comparison between the second form of the propagator, that we call $K_{2}$, and the first form $K_{1}$, it is convenient to break the time interval $T$ into $N-1$ intervals, rather than $N$. We write

$$
K_{2}\left(z^{\prime \prime}, T ; z^{\prime}, 0\right)=\left\langle z^{\prime \prime}\left|\prod_{j=1}^{N-1} \mathrm{e}^{-\frac{i \tau}{\hbar} \hat{H}}\right| z^{\prime}\right\rangle
$$

and, following Klauder and Skagerstam, we write the infinitesimal propagators as

$$
\mathrm{e}^{-\frac{i}{\hbar} \hat{H} \tau} \approx \int\left|z_{j}\right\rangle\left(1-\frac{i \tau}{\hbar} H_{2}\left(z_{j}^{\star}, z_{j}\right)\right) \frac{\mathrm{d}^{2} z_{j}}{\pi}\left\langle z_{j}\left|\approx \int\right| z_{j}\right\rangle \mathrm{e}^{-\frac{i \tau}{\hbar} H_{2}\left(z_{j}^{\star}, z_{j}\right)} \frac{\mathrm{d}^{2} z_{j}}{\pi}\left\langle z_{j}\right| .
$$

The complete propagator $K_{2}$ becomes

$$
\begin{aligned}
K_{2}\left(z_{N}, T\right. & \left.; z_{0}, 0\right)=\int \prod_{j=1}^{N-1} \frac{\mathrm{d}^{2} z_{j}}{\pi}\left\langle z_{j+1} \mid z_{j}\right\rangle \exp \left\{-\frac{i \tau}{\hbar} H_{2}\left(z_{j}^{\star}, z_{j}\right)\right\} \\
& =\int\left\{\prod_{j=1}^{N-1} \frac{\mathrm{d}^{2} z_{j}}{\pi}\right\} \exp \left\{\sum_{j=0}^{N-1}\left[\frac{1}{2}\left(z_{j+1}^{\star}-z_{j}^{\star}\right) z_{j}-\frac{1}{2} z_{j+1}^{\star}\left(z_{j+1}-z_{j}\right)-\frac{i \tau}{\hbar} H_{2}\left(z_{j}^{\star}, z_{j}\right)\right]\right\} .
\end{aligned}
$$

The differences between $K_{1}$ and $K_{2}$ are subtle but important. While the two arguments of $H_{1}$ in $K_{1}$ belong to two adjacent times in the mesh, the two arguments of $H_{2}$ in $K_{2}$ belong to the same time. Although both forms should give identical results 
when computed exactly, the differences between the two are important for the stationary exponent approximation, resulting in different semiclassical propagators. The semiclassical evaluation of $K_{1}$ and $K_{2}$ were presented in detail in [1]. Here we only list the results:

$$
\begin{aligned}
& K_{1}\left(z^{\prime \prime}, t ; z^{\prime}, 0\right)=\sum_{\nu} \sqrt{\frac{i}{\hbar} \frac{\partial^{2} S_{1 \nu}}{\partial u^{\prime} \partial v^{\prime \prime}}} \exp \left\{\frac{i}{\hbar}\left(S_{1 \nu}+I_{1 \nu}\right)-\frac{1}{2}\left(\left|z^{\prime \prime}\right|^{2}+\left|z^{\prime}\right|^{2}\right)\right\}, \\
& K_{2}\left(z^{\prime \prime}, t ; z^{\prime}, 0\right)=\sum_{\nu} \sqrt{\frac{i}{\hbar} \frac{\partial^{2} S_{2 \nu}}{\partial u^{\prime} \partial v^{\prime \prime}}} \exp \left\{\frac{i}{\hbar}\left(S_{2 \nu}-I_{2 \nu}\right)-\frac{1}{2}\left(\left|z^{\prime \prime}\right|^{2}+\left|z^{\prime}\right|^{2}\right)\right\},
\end{aligned}
$$

where

$$
S_{i \nu}=S_{i \nu}\left(v^{\prime \prime}, u^{\prime}, t\right)=\int_{0}^{t} \mathrm{~d} t^{\prime}\left[\frac{i \hbar}{2}(\dot{u} v-\dot{v} u)-H_{i}\left(u, v, t^{\prime}\right)\right]-\frac{i \hbar}{2}\left(u^{\prime \prime} v^{\prime \prime}+u^{\prime} v^{\prime}\right)
$$

is the action and

$$
I_{i}=\frac{1}{2} \int_{0}^{T} \frac{\partial^{2} H_{i}}{\partial u \partial v} \mathrm{~d} t
$$

is a correction to the action. The sum over $\nu$ represents the sum over all (complex) classical trajectories satisfying Hamilton's equations

$$
\begin{aligned}
i \hbar \dot{u} & =+\frac{\partial H_{i}}{\partial v} \\
i \hbar \dot{v} & =-\frac{\partial H_{i}}{\partial u}
\end{aligned}
$$

with boundary conditions

$$
u(0)=z^{\prime} \equiv u^{\prime}, \quad v(t)={z^{\prime \prime}}^{\star \star} \equiv v^{\prime \prime} .
$$

The factors $I_{i}$ are an important part of the above formulas and they are absolutely necessary to recover the exact propagator for quadratic Hamiltonians. If one neglects it, even the Harmonic oscillator comes out wrong. For a discussion about non-contributing trajectories, see refs. [16, 7].

\subsection{The Conjectured Weyl approximation}

As discussed at the begining of this section, for a given quantum operator $\hat{H}$, the first-form Hamiltonian is given by
$H_{1}\left(z^{\star}, z\right)=\langle z|\hat{H}| z\rangle$. It can be obtained by writing $\hat{H}$ in terms of the operators $\hat{a}$ and $\hat{a}^{\dagger}$ in normal order, so that each monomial in $\hat{H}$ look like $c_{n m} \hat{a}^{\dagger n} \hat{a}^{m}$. Replacing $\hat{a}^{\dagger}$ by $z^{\star}$ and $\hat{a}$ by $z$ yields $H_{1} . H_{2}$ can be calculated by writing $\hat{H}$ in anti-normal order, where now each monomial looks like $c_{n m} \hat{a}^{n} \hat{a}^{\dagger m}$, and then replacing $\hat{a}^{\dagger}$ by $z^{\star}$ and $\hat{a}$ by $z$. A third type of Hamiltonian function can be obtained from $\hat{H}$ by using the Wigner transformation:

$$
H_{W}(q, p)=\int \mathrm{d} s \mathrm{e}^{\frac{i}{\hbar} p s}\left\langle q-\frac{s}{2}|\hat{H}| q+\frac{s}{2}\right\rangle
$$

This is the Weyl Hamiltonian. Since $H_{W}$ is obtained from $\hat{H}$ by completely symmetrizing the creation and annihilation operators, it turns out that $H_{W}$ is an exact average between $H_{1}$ and $H_{2}$ if $\hat{H}$ contains up to cubic monomials in $\hat{a}$ and $\hat{a}^{\dagger}$, but only an approximate average for other cases. The semiclassical formula with $H_{1}$ comes with a correction $+I_{1}$ to the action and that with $H_{2}$ comes with a correction of $-I_{2}$. This suggests a third type of semiclassical approximation for the propagator, where one uses the Weyl Hamiltonian and no correction term, since the average of $+I_{1}$ and $-I_{2}$ should be approximately zero. This is the Weyl approximation, which was conjecture in [1]:

$$
K_{W}\left(z^{\prime \prime}, t ; z^{\prime}, 0\right)=\sum_{\nu} \sqrt{\frac{i}{\hbar} \frac{\partial^{2} S_{W}}{\partial u^{\prime} \partial v^{\prime \prime}}} \exp \left\{\frac{i}{\hbar} S_{W}-\frac{1}{2}\left(\left|z^{\prime \prime}\right|^{2}+\left|z^{\prime}\right|^{2}\right)\right\}
$$

with $S_{W}$ given by Eq.(17) with $H_{i}$ replaced by $H_{W}$.

Of the three semiclassical approximations presented, the Weyl approximation seems to be the most natural, since it involves the classical hamiltonian directly and no correc- tions to the action. However, this formula does not follow from the two most natural forms of path integral proposed by Klauder and used in this section. In the next section we propose a third form of path integral whose semiclassical 
limit is indeed the Weyl approximation.

\section{A Mixed Form for the Path Integral}

The new form of path integral we describe in this section is based on the fact that $H_{W}$ is almost the average of $H_{1}$ and $H_{2}$. The idea is to force this average to appear by combining the first and second form of path integrals in alternating time steps. We start from

$$
K\left(z^{\prime \prime}, t ; z^{\prime}, 0\right)=\left\langle z_{N}\left|\prod_{j=0}^{N-1} \mathrm{e}^{-\frac{i}{\hbar} \hat{H} \tau_{j}}\right| z_{0}\right\rangle
$$

where $z_{N}=z^{\prime \prime}, z_{0}=z^{\prime}, \tau_{j}$ is the time step and we take $N$ to be even for convenience. Although we shall consider the time steps $\tau_{j}$ to be all equal later, we keep the index $j$ for now to keep track of the time intervals.

For j odd we approximate

$$
\mathrm{e}^{-\frac{i}{\hbar} \hat{H} \tau_{j}} \approx \int\left|z_{j}\right\rangle\left(1-\frac{i \tau_{j}}{\hbar} H_{2}\left(z_{j}^{\star}, z_{j}\right)\right) \frac{\mathrm{d}^{2} z_{j}}{\pi}\left\langle z_{j}\left|\approx \int\right| z_{j}\right\rangle \mathrm{e}^{-\frac{i \tau_{j}}{\hbar} H_{2}\left(z_{j}^{\star}, z_{j}\right)} \frac{\mathrm{d}^{2} z_{j}}{\pi}\left\langle z_{j}\right|
$$

For $j>0$ even we simply insert a unit operator on the right of the infinitesimal propagator:

$$
\mathrm{e}^{-\frac{i}{\hbar} \hat{H} \tau_{j}}=\int \mathrm{e}^{-\frac{i}{\hbar} \hat{H} \tau_{j}}\left|z_{j}\right\rangle \frac{\mathrm{d}^{2} \mathrm{z}_{\mathrm{j}}}{\pi}\left\langle z_{j}\right|
$$

Multiplying this operator on the left by the bra $\left\langle z_{j-1}\right|$ coming from the odd term $j-1$ and using the approximation employed in the first form of path integrals, Eq.(8), we get the following mixed form for the propagator:

$$
\begin{aligned}
K\left(z_{N}, T ; z_{0}, 0\right) & =\int\left\{\prod_{j=1}^{N-1} \frac{\mathrm{d}^{2} z_{j}}{\pi}\right\} \exp \left\{\sum _ { j = 0 } ^ { N - 1 } \left[\frac{1}{2}\left(z_{j+1}^{\star}-z_{j}^{\star}\right) z_{j}-\frac{1}{2} z_{j+1}^{\star}\left(z_{j+1}-z_{j}\right)\right.\right. \\
& \left.\left.-\frac{i \tau}{\hbar} a_{j} H_{2, j}-\frac{i \tau}{\hbar} b_{j} H_{1, j}\right]\right\} \\
& \equiv \int\left\{\prod_{j=1}^{N-1} \frac{\mathrm{d} z_{j}^{\star} \mathrm{d} z_{j}}{2 \pi i}\right\} \mathrm{e}^{f\left(z^{\star}, z\right)}
\end{aligned}
$$

where $a_{j}$ is zero for $j$ even and one for $j$ odd, $b_{j}$ is zero for $j$ odd and one for $j$ is even. At this point we have suppressed the index on the time intervals and have taken $\tau_{j}=\tau$. The exponent $f$ is given by

$$
f\left(z^{\star}, z\right)=\sum_{j=0}^{N-1}\left\{\frac{1}{2}\left(z_{j+1}^{\star}-z_{j}^{\star}\right) z_{j}-\frac{1}{2} z_{j+1}^{\star}\left(z_{j+1}-z_{j}\right)-\frac{i \tau}{\hbar} a_{j} H_{2, j}-\frac{i \tau}{\hbar} b_{j} H_{1, j}\right\}
$$

where we have introduced the abbreviated notation $H_{2, j} \equiv H_{2}\left(z_{j}^{\star}, z_{j}\right)$ and $H_{1, j} \equiv \mathcal{H}\left(z_{j+1}^{*}, z_{j}\right)$.

\subsection{The Stationary Exponent Approximation}

In the semiclassical limit $\hbar \rightarrow 0$ we can approximate the integrals (26) by looking for the places where the exponent $f$ is stationary and replacing it in their vicinity by a quadratic form of its variables $\left(z^{\star}, z\right)$. We find the stationary points by requiring the vanishing of the derivatives of $f$ with respect to $z$ and $z^{\star}$ separately. We obtain

$$
\begin{array}{ccrl}
\frac{\partial f}{\partial z_{j}}=z_{j+1}^{\star}-z_{j}^{\star}-\frac{i a_{j} \tau}{\hbar} \frac{\partial H_{2, j}}{\partial z_{j}}-\frac{i b_{j} \tau}{\hbar} \frac{\partial H_{1, j}}{\partial z_{j}} \quad=0 ; & j=1, \ldots, N-1 \\
\frac{\partial f}{\partial z_{j+1}^{\star}}=-z_{j+1}+z_{j}-\frac{i a_{j+1} \tau}{\hbar} \frac{\partial H_{2, j+1}}{\partial z_{j+1}^{\star}}-\frac{i b_{j} \tau}{\hbar} \frac{\partial H_{1, j}}{\partial z_{j+1}^{\star}}=0 ; & j=0, \ldots, N-2 .
\end{array}
$$

We now introduce new integration variables $\eta$ and $\eta^{\star}$, which describe the deviations from the points of stationary exponent, $z \rightarrow z+\eta, z^{\star} \rightarrow z^{\star}+\eta^{\star}$, with the boundary conditions

$$
\eta_{0}=\eta_{0}^{\star}=\eta_{N}=\eta_{N}^{\star}=0
$$


Expanding the exponent into a Taylor series in $\left(\eta^{\star}, \eta\right)$ around the stationary points $\left(z^{\star}, z\right)$ up to second order and re-inserting the result into (6) yields

$$
\begin{aligned}
K\left(z^{\prime \prime}, t ; z^{\prime}, 0\right)=\mathrm{e}^{f\left(z^{\star}, z\right)} \int\left\{\prod_{j=1}^{N-1} \frac{\mathrm{d} \eta_{j}^{\star} \mathrm{d} \eta_{j}}{2 \pi i}\right\} \exp \sum_{j=0}^{N-1}\left\{-\frac{i \tau}{2 \hbar}\left[b_{j} \frac{\partial^{2} H_{1, j}}{\partial z_{j}^{2}}+a_{j} \frac{\partial^{2} H_{2, j}}{\partial z_{j}^{2}}\right] \eta_{j}^{2}\right. \\
-\frac{i}{2 \hbar}\left[b_{j} \tau \frac{\partial^{2} H_{1, j}}{\partial z_{j+1}^{\star 2}}+a_{j+1} \tau \frac{\partial^{2} H_{2, j+1}}{\partial z_{j+1}^{\star 2}}\right] \eta_{j+1}^{\star 2} \\
\left.-\left(1+\frac{i \tau a_{j}}{\hbar} \frac{\partial^{2} H_{2, j}}{\partial z_{j}^{\star} \partial z_{j}}\right) \eta_{j}^{\star} \eta_{j}+\left(1-\frac{i \tau b_{j}}{\hbar} \frac{\partial^{2} H_{1, j}}{\partial z_{j+1}^{\star} \partial z_{j}}\right) \eta_{j+1}^{\star} \eta_{j}\right\} .
\end{aligned}
$$

The integrals in Eq.(30) can be carried out using the same techniques presented in [1]. The idea is to integrate first over $\eta_{1}^{\star}$ and $\eta_{1}$, then over $\eta_{2}^{\star}$ and $\eta_{2}$, etc. A recursion formula can be readily established and, once all integrations are done, we obtain

$$
K\left(z_{N}, t ; z_{0}, 0\right)=\mathrm{e}^{f\left(z^{\star}, z\right)} \prod_{j=1}^{N-1} \frac{1}{\sqrt{\left(1+\frac{i \tau a_{j}}{\hbar} \frac{\partial^{2} H_{2, j}}{\partial z_{j}^{\star} \partial z_{j}}\right)^{2}+2 i \frac{\tau}{\hbar}\left(a_{j} \frac{\partial^{2} H_{2, j}}{\partial z_{j}^{2}}+b_{j} \frac{\partial^{2} H_{1, j}}{\partial z_{j}^{2}}\right) X_{j}}}
$$

where $X_{j}$ satisfies

$$
\begin{aligned}
& X_{j}=-\frac{i \tau a_{j}}{2 \hbar} \frac{\partial^{2} H_{2, j}}{\partial z_{j}^{\star 2}}-\frac{i \tau b_{j-1}}{2 \hbar} \frac{\partial^{2} H_{1, j-1}}{\partial z_{j}^{\star 2}} \\
& +\frac{\left(1-\frac{i \tau b_{j-1}}{\hbar} \frac{\partial^{2} H_{1, j-1}}{\partial z_{j}^{\star} \partial z_{j-1}}\right)^{2}}{\left(1+\frac{i \tau a_{j-1}}{\hbar} \frac{\partial^{2} H_{2, j-1}}{\partial z_{j-1}^{\star} \partial z_{j-1}}\right)^{2}+2 \frac{i \tau}{\hbar}\left(a_{j-1} \frac{\partial^{2} H_{2, j-1}}{\partial z_{j-1}^{2}}+b_{j-1} \frac{\partial^{2} H_{1, j-1}}{\partial z_{j-1}^{2}}\right) X_{j-1}} X_{j-1}
\end{aligned}
$$

for $j=1, \ldots, N-1$ with $X_{0}=0$.

\subsection{The Effective Hamiltonian}

To obtain the continuum limit of the discrete equations of motion (28) we first note that, since $a_{j}$ is zero for $j$ even and one for $j$ odd, and $b_{j}$ is zero for $j$ odd and one for $j$ is even, the second of Eqs.(28) gives

$$
-z_{j+1}+z_{j}=0
$$

for $j$ even.

This result motivates the choice of a new time step $\epsilon=2 \tau$, in such a way that the discretized time evolution goes from $z_{j}$ directly to $z_{j+2}$ for $j$ even and from $z_{j}^{\star}$ directly to $z_{j+2}^{\star}$ for $j$ odd . This choice of time step makes sense if one can find an effective Hamiltonian able to perform the corresponding evolution for both variables $z$ and $z^{*}$. Indeed, the equations of motion can be put the form

$$
\begin{array}{ll}
\frac{z_{j+1}-z_{j-1}}{\epsilon}=-\frac{i}{\hbar} \frac{\partial H_{e f, j}}{\partial z_{j}^{\star}} & j=1,3,5 \ldots, N-3 \\
\frac{z_{j+1}^{\star}-z_{j-1}^{\star}}{\epsilon}=\frac{i}{\hbar} \frac{\partial H_{e f, j}}{\partial z_{j}} & j=2,4, \ldots, N-2 .
\end{array}
$$

where

$$
\begin{aligned}
H_{e f, j} & \equiv\left(b_{j} H_{1, j}+b_{j-1} H_{1, j-1}+a_{j-1} H_{2, j}+a_{j} H_{2, j+1}\right) / 2 \\
& = \begin{cases}\frac{H_{1, j}+H_{2, j}}{2} & \text { for } j \text { even } \\
\frac{H_{1, j-1}+H_{2, j+1}}{2} & \text { for } j \text { odd } .\end{cases}
\end{aligned}
$$

Note that, because we are skipping points with the new time step $\epsilon=2 \tau$, we miss the point $z_{N}^{\star}$ in Eq.(35). This, however, does not affect the limit of the continuum, as long as we take $z_{N-1}^{\star}=z_{N}^{\star}$ to ensure the proper boundary condition.

In the limit where $2 \tau=\epsilon$ goes to zero the effective Hamiltonian reduces to

$$
\mathcal{H}_{\mathcal{C}}\left(z^{\star}, z\right) \equiv \frac{H_{1}\left(z^{\star}, z\right)+H_{2}\left(z^{\star}, z\right)}{2} .
$$

As in the case of the semiclassical formulas Eqs.(15) and (16) for $K_{1}$ and $K_{2}$, the stationary trajectory is usually complex. It is therefore convenient to follow the notation intro- 
duced in [1] and make the substitutions

$$
\begin{aligned}
z \rightarrow u & =\frac{1}{\sqrt{2}}\left(\frac{q}{b}+i \frac{p}{c}\right) \\
z^{\star} \rightarrow v & =\frac{1}{\sqrt{2}}\left(\frac{q}{b}-i \frac{p}{c}\right) .
\end{aligned}
$$

In terms of $u$ and $v$, the stationary phase conditions (28) turn into Hamiltons equations with $\mathcal{H}_{\mathcal{C}}$ :

$$
\begin{aligned}
i \hbar \dot{u} & =+\frac{\partial \mathcal{H}_{\mathcal{C}}}{\partial v} \\
i \hbar \dot{v} & =-\frac{\partial \mathcal{H}_{\mathcal{C}}}{\partial u}
\end{aligned}
$$

with boundary conditions identical to Eq.(20). The function $f$ can also be simplified to

$$
f=\int_{0}^{t} \mathrm{~d} t^{\prime}\left[\frac{1}{2}(\dot{v} u-\dot{u} v)-\frac{i}{\hbar} \mathcal{H}_{\mathcal{C}}\left(u, v, t^{\prime}\right)\right]+\frac{1}{2}\left(v^{\prime \prime} u^{\prime \prime}+v^{\prime} u^{\prime}\right)-\frac{1}{2}\left(\left|z^{\prime \prime}\right|^{2}+\left|z^{\prime}\right|^{2}\right)
$$

where $u(0)=u^{\prime}=z^{\prime}, v(0)=v^{\prime}, u(T)=u "$ and $v(T)=v "=z^{\prime \prime}$.

Next we calculate the product appearing in (31). Performing the limit $N \rightarrow \infty$ and using the expansion $\ln (1+x)=$ $x+O\left(x^{2}\right)$ we obtain

$$
\begin{aligned}
\Gamma \equiv & \lim _{N \rightarrow \infty} \prod_{j=1}^{N-1}\left\{\left(1+\frac{i \tau a_{j}}{\hbar} \frac{\partial^{2} H_{2, j}}{\partial z_{j}^{\star} \partial z_{j}}\right)^{2}+2 i \frac{\tau}{\hbar}\left(a_{j} \frac{\partial^{2} H_{2, j}}{\partial z_{j}^{2}}+b_{j} \frac{\partial^{2} H_{1, j}}{\partial z_{j}^{2}}\right) X_{j}\right\}^{-\frac{1}{2}} \\
& =\lim _{N \rightarrow \infty} \exp \left\{-\frac{1}{2} \sum_{j=1}^{N-1} \ln \left[1+\frac{2 i \tau a_{j}}{\hbar} \frac{\partial^{2} H_{2, j}}{\partial z_{j}^{\star} \partial z_{j}}+2 i \frac{\tau}{\hbar}\left(a_{j} \frac{\partial^{2} H_{2, j}}{\partial z_{j}^{2}}+b_{j} \frac{\partial^{2} H_{1, j}}{\partial z_{j}^{2}}\right) X_{j}+O\left(\tau^{2}\right)\right]\right\} \\
& =\lim _{N \rightarrow \infty} \exp \left\{-\frac{i}{\hbar} \sum_{j=1}^{N-1} \tau\left[a_{j} \frac{\partial^{2} H_{2, j}}{\partial z_{j}^{\star} \partial z_{j}}+\left(a_{j} \frac{\partial^{2} H_{2, j}}{\partial z_{j}^{2}}+b_{j} \frac{\partial^{2} H_{1, j}}{\partial z_{j}^{2}}\right) X_{j}\right]\right\} .
\end{aligned}
$$

In order to transform these sums into integrals we note that

$$
\begin{aligned}
\lim _{N \rightarrow \infty}\left\{\sum_{j=1}^{N-1} \tau\left(a_{j} F\left(t_{j}\right)\right)\right\} & =\lim _{N \rightarrow \infty} \tau\left(F\left(t_{1}\right)+F\left(t_{3}\right)+F\left(t_{5}\right)+\ldots\right) \\
& =\frac{1}{2} \lim _{N \rightarrow \infty} \tau\left(F\left(t_{1}\right)+F\left(t_{1}\right)+F\left(t_{3}\right)+F\left(t_{3}\right)+F\left(t_{5}\right)+\ldots\right) \\
& =\frac{1}{2} \lim _{N \rightarrow \infty} \tau\left(F\left(t_{1}\right)+F\left(t_{2}\right)+F\left(t_{3}\right)+F\left(t_{4}\right)+F\left(t_{5}\right)+\ldots\right) \\
& =\frac{1}{2} \int_{0}^{t} \mathrm{~d} t^{\prime} F\left(t^{\prime}\right)
\end{aligned}
$$

since, for smooth functions, $F\left(t_{j}\right) \rightarrow F\left(t_{j+1}\right)$ as $\tau \rightarrow 0$. The integrals with the coefficients $b_{j}$ also acquire the $1 / 2$ factor. Using these results we obtain

$$
\begin{aligned}
\Gamma= & =\exp \left\{-\frac{i}{\hbar} \int_{0}^{t} \mathrm{~d} t^{\prime}\left(\frac{1}{2} \frac{\partial^{2} H_{2}}{\partial u \partial v}\left(t^{\prime}\right)+\frac{1}{2}\left(\frac{\partial^{2} H_{1}}{\partial u^{2}}+\frac{\partial^{2} H_{2}}{\partial u^{2}}\right) X\left(t^{\prime}\right)\right)\right\} \\
& =\exp \left\{-\frac{i}{\hbar} \int_{0}^{t} \mathrm{~d} t^{\prime}\left[\frac{1}{2} \frac{\partial^{2} H_{2}}{\partial u \partial v}\left(t^{\prime}\right)+\frac{\partial^{2} \mathcal{H}_{\mathcal{C}}}{\partial u^{2}}\left(t^{\prime}\right) X\left(t^{\prime}\right)\right]\right\} .
\end{aligned}
$$

\subsection{The Effective Phase}

Replacing $\Gamma$ and $f$ into Eq.(31) we obtain

$$
\begin{aligned}
K\left(z^{\prime \prime}, t ; z^{\prime}, 0\right) & =\exp \left\{-\frac{i}{\hbar} \int_{0}^{t} \mathrm{~d} t^{\prime}\left[\frac{1}{2} \frac{\partial^{2} \mathcal{H}_{2}}{\partial u \partial v}\left(t^{\prime}\right)+\frac{\partial^{2} \mathcal{H}_{\mathcal{C}}}{\partial u^{2}}\left(t^{\prime}\right) X\left(t^{\prime}\right)\right]\right\} \\
& \exp \left\{\int_{0}^{t} \mathrm{~d} t^{\prime}\left[\frac{1}{2}(\dot{v} u-\dot{u} v)-\frac{i}{\hbar} \mathcal{H}_{\mathcal{C}}\right]+\frac{1}{2}\left(v^{\prime} u^{\prime}+v^{\prime \prime} u^{\prime \prime}\right)-\frac{1}{2}\left(\left|z^{\prime}\right|^{2}+\left|z^{\prime \prime}\right|^{2}\right)\right\} .
\end{aligned}
$$


We still have to write the continuous form of the discrete recursion formula (32) for $X(t)$. In the limit $N \rightarrow \infty$ we obtain the nonlinear differential equation

$$
\dot{X}(t)=-\frac{i}{2 \hbar} \frac{\partial^{2} \mathcal{H}_{\mathcal{C}}}{\partial v^{2}}-2 \frac{i}{\hbar} \frac{\partial^{2} \mathcal{H}_{\mathcal{C}}}{\partial u \partial v} X(t)-2 \frac{i}{\hbar} \frac{\partial^{2} \mathcal{H}_{\mathcal{C}}}{\partial u^{2}} X^{2}(t)
$$

with the initial condition $X(0)=0$.

This equation was solved in [1] and the result is $X=\frac{1}{2} \frac{\delta u}{\delta v}$ where $\delta u$ and $\delta v$ are solutions of the linearized Hamilton's equations

$$
\begin{aligned}
\delta \dot{u} & =-\frac{i}{\hbar} \frac{\partial^{2} \mathcal{H}_{\mathcal{C}}}{\partial u \partial v} \delta u-\frac{i}{\hbar} \frac{\partial^{2} \mathcal{H}_{\mathcal{C}}}{\partial v^{2}} \delta v \\
\delta \dot{v} & =+\frac{i}{\hbar} \frac{\partial^{2} \mathcal{H}_{\mathcal{C}}}{\partial u^{2}} \delta u+\frac{i}{\hbar} \frac{\partial^{2} \mathcal{H}_{\mathcal{C}}}{\partial u \partial v} \delta v
\end{aligned}
$$

where the derivatives are calculated at the stationary trajectory and the initial conditions are $\delta u(0)=0$ and $\delta v(0)$ arbitrary. The second term in the first exponential of (44) can be now transformed with the help of (46)

$$
\frac{i}{\hbar} \frac{\partial^{2} \mathcal{H}_{\mathcal{C}}}{\partial u^{2}} X=\frac{i}{2 \hbar} \frac{\partial^{2} \mathcal{H}_{\mathcal{C}}}{\partial u^{2}} \frac{\delta u}{\delta v}=\frac{1}{2} \frac{\delta \dot{v}}{\delta v}-\frac{i}{2 \hbar} \frac{\partial^{2} \mathcal{H}_{\mathcal{C}}}{\partial u \partial v}=\frac{1}{2} \frac{\mathrm{d}}{\mathrm{dt}} \ln \delta v-\frac{i}{2 \hbar} \frac{\partial^{2} \mathcal{H}_{\mathcal{C}}}{\partial u \partial v}
$$

so that the first exponent of (44) becomes

$$
\begin{aligned}
& \exp \left\{-\frac{i}{\hbar} \int_{0}^{t} \mathrm{~d} t^{\prime}\left[\frac{1}{2} \frac{\partial^{2} H_{2}}{\partial u \partial v}\left(t^{\prime}\right)+\frac{\partial^{2} \mathcal{H}_{\mathcal{C}}}{\partial u^{2}}\left(t^{\prime}\right) X\left(t^{\prime}\right)\right]\right\} \\
& =\exp \left\{+\frac{i}{4 \hbar} \int_{0}^{t} \mathrm{~d} t^{\prime}\left[\frac{\partial^{2} \mathcal{H}_{1}}{\partial u \partial v}\left(t^{\prime}\right)-\frac{\partial^{2} \mathcal{H}_{2}}{\partial u \partial v}\left(t^{\prime}\right)\right]\right\} \exp \left\{-\frac{1}{2} \int_{0}^{t} \mathrm{~d} t^{\prime}\left[\frac{\mathrm{d}}{\mathrm{d} t^{\prime}}(\ln \delta v)\right]\right\} \\
& =\sqrt{\frac{\delta v^{\prime}}{\delta v^{\prime \prime}}} \exp \left[\frac{i}{\hbar}\left(\frac{I_{1}-I_{2}}{2}\right)\right] \equiv \sqrt{\frac{\delta v^{\prime}}{\delta v^{\prime \prime}}} \exp \left[\frac{i}{\hbar} I_{C}\right] .
\end{aligned}
$$

The pre-factor $\delta v^{\prime} / \delta v^{\prime \prime}$ can also be written in terms of the action using $\partial S_{C} / \partial u^{\prime}=-i \hbar v^{\prime}$, where

$$
S_{C}\left(v^{\prime \prime}, u^{\prime}, t\right):=\int_{0}^{t} \mathrm{~d} t^{\prime}\left[\frac{i \hbar}{2}(\dot{u} v-\dot{v} u)-H_{C}\left(u, v, t^{\prime}\right)\right]-\frac{i \hbar}{2}\left(u^{\prime \prime} v^{\prime \prime}+u^{\prime} v^{\prime}\right)
$$

is the effective action. In the end we obtain

$$
K\left(z^{\prime \prime}, t ; z^{\prime}, 0\right)=\sqrt{\frac{i}{\hbar} \frac{\partial^{2} S_{C}}{\partial u^{\prime} \partial v^{\prime \prime}}} \exp \left\{\frac{i}{\hbar}\left(S_{C}+I_{C}\right)-\frac{1}{2}\left(\left|z^{\prime}\right|^{2}+\left|z^{\prime \prime}\right|^{2}\right)\right\}
$$

where

$$
I_{C}=\frac{1}{2}\left(I_{1}-I_{2}\right)
$$

is the effective phase.

\section{The Weyl Approximation}

Equation (51) is the main result of this paper. It represents a third semiclassical approximation for the coherent states propagator, involving the effective Hamiltonian $H_{C}=$ $\left(H_{1}+H_{2}\right) / 2$ and the effective phase $I_{C}=\left(I_{1}-I_{2}\right) / 2$.

For the harmonic oscillator $I_{1}$ and $I_{2}$ are exactly equal and $I_{C}=0$. In this case $H_{C}$ coincides with the classical, or Weyl, Hamiltonian $H_{W}$, and the conjectured Weyl approximation (22) is obtained. In fact $I_{1}=I_{2}$ and $H_{C}=H_{W}$ for all polynomial Hamiltonians involving up to cubic powers of $q$ or $p$ [1]. This can be seen from the formulas [1, 2]

$$
H_{1}\left(z^{\star}, z\right)=\exp \left(\frac{1}{2} \hat{\delta}\right) H_{W}\left(z^{\star}, z\right)
$$

$$
H_{2}\left(z^{\star}, z\right)=\exp \left(-\frac{1}{2} \hat{\delta}\right) H_{W}\left(z^{\star}, z\right)
$$


where $\hat{\delta}=\partial^{2} / \partial z^{\star} \partial z$. This gives

$$
H_{C}=\cosh \left(\frac{1}{2} \hat{\delta}\right) H_{W}=H_{W}-\frac{1}{8} \frac{\partial^{4}}{\partial z^{2} \partial z^{\star 2}} H_{W}+\ldots
$$

which shows explicitly that $H_{C}=H_{W}$ for up to cubic polynomials. Besides, using the relations

$$
q=\left(z+z^{\star}\right)\left(\frac{b}{\sqrt{2}}\right) \quad p=\left(z-z^{\star}\right)\left(\frac{-i \hbar}{b \sqrt{2}}\right)
$$

with $b \sim c \sim O\left(\hbar^{1 / 2}\right)$, we see that quartic or higher order terms contribute to $H_{C}$ or $I_{C}$ only terms of order $\hbar^{2}$. These terms can in principle be neglected, since they are beyond the scope of the approximation. With these considerations we can rewrite the propagator (50) as

$$
K\left(z^{\prime \prime}, t ; z^{\prime}, 0\right)==\sqrt{\frac{i}{\hbar} \frac{\partial^{2} S_{W}}{\partial u^{\prime} \partial v^{\prime \prime}}} \exp \left\{\frac{i}{\hbar} S_{W}-\frac{1}{2}\left(\left|z^{\prime}\right|^{2}+\left|z^{\prime \prime}\right|^{2}\right)\right\}
$$

where $S_{W}$ is given by Eq. (50) with $H_{C}$ replaced by $H_{W}$. This is the Weyl formula conjectured in [1].

As a final remark we notice that the differences between the three semiclassical formulas presented in this paper are of the order of $\hbar$, and go to zero in the semiclassical limit. These differences, however, are always relevant at low energies, and can be made explicit by considering the Fourier transform of these time dependent formulas. A discussion of the energy representation of these semiclassical propagators were presented in section 6 of ref.[1], including the derivation of semiclassical quantization rules, and we refer to it for further details.

\section{Acknowledgments}

MAMA acknowledges financial support from the Brazilian agencies FAPESP and CNPq.

\section{References}

[1] M. Baranger, M. A. M. de Aguiar, F. Keck, H. J. Korsch, and B. Schellhaa, J. Phys. A 34, 7227 (2001).

[2] J. R. Klauder and B. S. Skagerstam, Coherent States, Applications in Physics and Mathematical Physics, World Scientific, Singapore, 1985.

[3] J. R. Klauder, Continuous Representations and Path Integrals, Revisited, in G. J. Papadopoulos and J. T. Devreese, editors, Path Integrals, NATO Advanced Study Institute, Series B: Physics, page 5, New York, 1978. Plenum.
[4] J. R. Klauder, Phys. Rev. D 19(8), 2349 (1979).

[5] J. R. Klauder, Some Recent Results on Wave Equations, Path Integrals and Semiclassical Approximations, in G. Papanicolaou, editor, Random Media, Random Media. Springer, 1987.

[6] Y. Weissman, J. Chem. Phys. 76, 4067 (1982).

[7] S. Adachi, Ann. of Phys., 195, 45 (1989).

[8] A. Shudo and K.S. Ikeda, Phys. Rev. Lett., 74, 682 (1995).

[9] A. L. Xavier, Jr. and M. A. M. de Aguiar, Ann. Phys. (N.Y.) 252, 458 (1996); A. L. Xavier, Jr. and M. A. M. de Aguiar, Phys. Rev. A 54(3), 1808 (1996); A. L. Xavier, Jr. and M. A. M. de Aguiar, Phys. Rev. Lett. 79(18), 3323 (1997).

[10] F. Grossmann and Jr. A. L. Xavier, Phys. Lett. A 243, 243 (1998).

[11] J. L. Schoendorff, H. J. Korsch, and N. Moiseyev, Europhys. Lett. 44, 290 (1998).

[12] T. Van Voorhis and Eric J. Heller, Phys. Rev. A 66, 050501 (2002).

[13] T. Van Voorhis and Eric J. Heller, J. Chem. Phys. 119, 12153 (2003).

[14] E. Pollak and J. Shao, J. Phys. Chem. A 107, 7112 (2003).

[15] F. Parisio and M.A.M. de Aguiar, Phys. Rev. A 68, 62112 (2003).

[16] A. D. Ribeiro, M. A. M. de Aguiar, and M. Baranger, Phys. Rev. E 69, 66204 (2004).

[17] K. M. Fonseca Romero, M.C. Nemes, J.G. Peixoto de Faria, and A.F.R. de Toledo Piza, Phys. Lett. A 327, 129 (2004).

[18] M. Hillery, R. F. O‘Connel, M. O. Scully, and E. P. Wigner, Phys. Rep. 106, 121 (1984).

[19] A.M. Ozorio de Almeida, Phys. Rep. 295, 265 (1998). 